\title{
Primary Intracranial Mucosa-Associated Lymphoid Tissue Lymphoma A Report of Two Cases and Literature Review
}

\author{
CRISTINA SEBASTIÁN ${ }^{1}$, ANA CARMEN VELA ${ }^{1}$, RAMÓN FIGUEROA² ${ }^{2}$ MIGUEL ÁNGEL MARÍN \\ JORGE ALFARO ${ }^{1}$ \\ ${ }^{1}$ Department of Radiology, Regional Hospital; Kemerovo, Russia; Department of Radiology, University Hospital Miguel \\ Servet; Zaragoza, Spain \\ ${ }^{2}$ Georgia Regents Medical Center; Georgia,USA
}

Key words: mucosa-associated lymphoid tissue lymphoma, B cell MALT lymphoma, low grade lymphoma, choroid plexus lymphoma, intracranial lymphoma, dural-based lymphoma

SUMMARY - Low-grade B cell non-Hodgkin lymphomas typically arise from the marginal zone of the secondary lymphatic follicles. Their intracranial expression is very rare, most frequently affecting the dura mater and the choroid plexus glomi in the lateral ventricles. Their initial evaluation requires the exclusion of more common extra-axial lesions, such as meningiomas, dural metastasis, granulomatous lesions or secondary lymphoproliferative dural extension from body lymphomas. Whenever a ventricular lesion is present, the patient's age and lesion location help narrow the differential diagnosis. Dural-based lymphomas and ventricular/choroid plexus lymphomas are slowgrowing lesions with imaging features similar to meningiomas, which is typically their main differential consideration. Diffusion-weighted images frequently show restricted diffusion behaviour on lymphomas, helping to differentiate them from the typical meningiomas.

\section{Introduction}

Mucosal-associated lymphoid tissue ("MALT") lymphomas originate from B-cell lymphocytes in the marginal zone of the secondary lymphatic follicles, representing only about $7 \%$ of the non-Hodgkin lymphomas ${ }^{1}$. These cells preferentially affect large lymphatic organs, such as the spleen and large lymph nodes. They can also affect some non-lymphatic organs involving small lymphoid tissue clusters in mucosal and non-mucosal layers, such as skin, orbit or dura mater.

MALT lymphomas were initially described in the stomach, the most frequently affected extranodal organ, as low-grade lymphomas associated with the lymphoid tissue contained within the organ mucosa, frequently related to the chronic inflammation associated with Helicobacter pylori, a common offending gastric bacteria ${ }^{1}$. The second most frequent extranodal site is the Waldeyer ring of the nasopharynx. Subsequent descriptions of MALT lymphomas affecting other sites were published including lung, gallbladder, salivary glands, conjunctival and lacrimal gland mucosa ${ }^{1,2}$. An etiologic relationship with immune system diseases is suspected in some territories, especially in lacrimal glands affected by Sjögren syndrome 2. Additional reports determined that MALT lymphomas could also originate from epithelial structures without apparent mucosal substrate, such as thyroid gland, thymus, breast, liver, orbit and skin ${ }^{1-3}$. MALT lymphomas are thus considered a localized variant of general, low-grade indolent lymphomas, with usually a clinically favourable prognosis ${ }^{4}$. We describe two cases of rare intracranial MALT lymphoma from our institutional experience.

\section{Case 1}

A 51-year-old man was seen through the emergency room due to new onset of a partial tonic epileptic seizure starting with right 


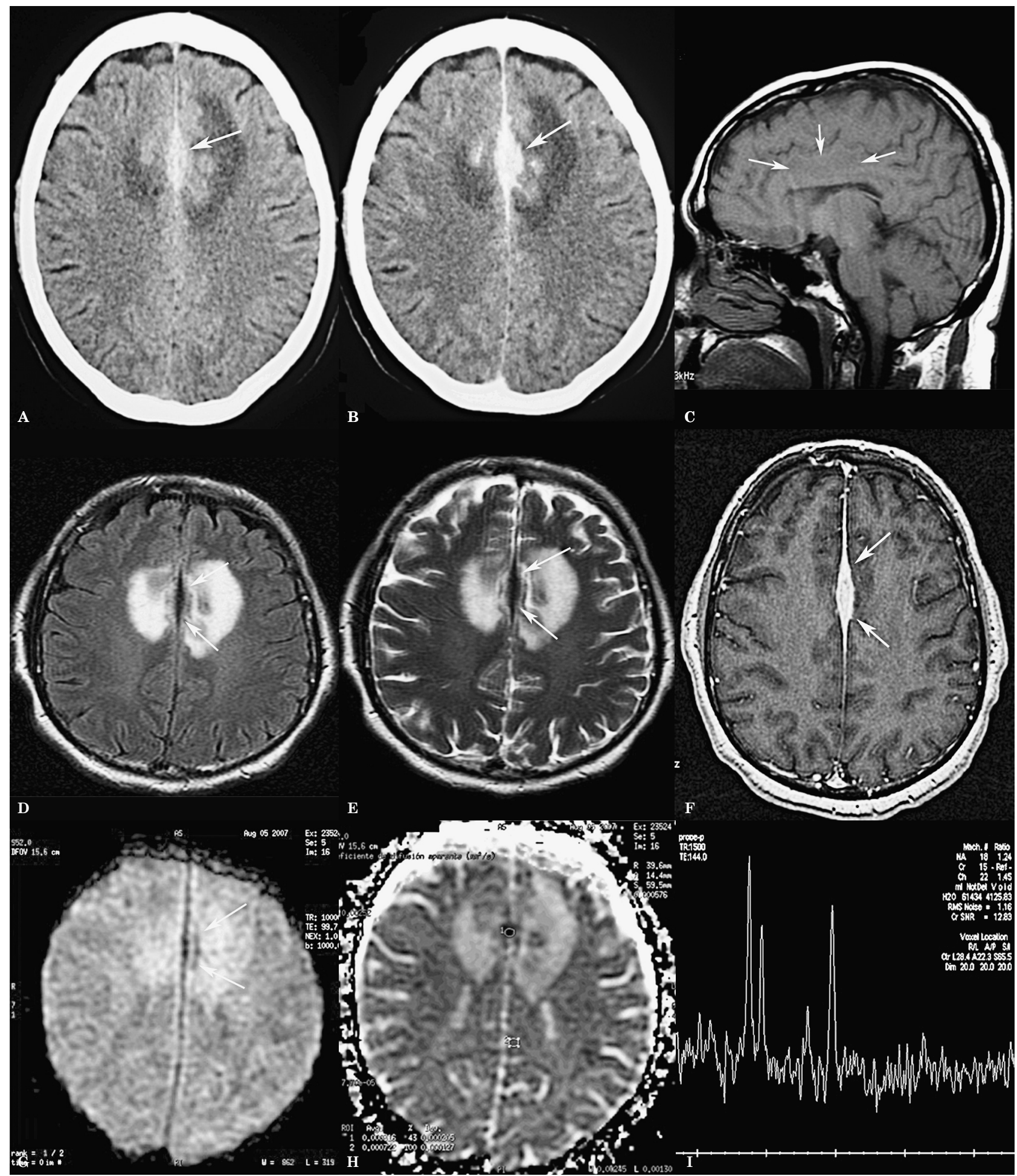

Figure 1 Case 1. A,B) Head CT without and with contrast show a hyperdense extra-axial lesion within the interhemispheric fissure with associated bilateral frontal subcortical vasogenic oedema and intense homogeneous enhancement after intravenous contrast. Magnetic resonance imaging (MRI) evaluation shows a T1 and FLAIR isointense lesion to grey matter (C,D) with T2 hypointense behaviour (E) and perilesional oedema. F) The gadolinium-enhanced axial T1 image shows homogeneously intense enhancement. G) The diffusion-weighted image shows lesion isointensity, while the apparent diffusion coefficient map $(\mathrm{H})$ shows moderate diffusion restriction (ROI 1) compared to normal grey matter (ROI 2). I) Proton MR Spectroscopy shows a significant elevation of the choline peak with a choline/creatine ratio larger than 1, a mild decrease in N-acetyl-aspartate (NAA), and no lipid or lactate abnormalities. J,K) Follow-up MRI show a significant reduction in perilesional oedema, with the lesion borders also reduced in size, becoming less defined. L) Pathology shows a massive lymphoid cell infiltration, composed of predominantly small lymphoid cells with few plasmatic cells and larger cells with vesicular nuclei. 

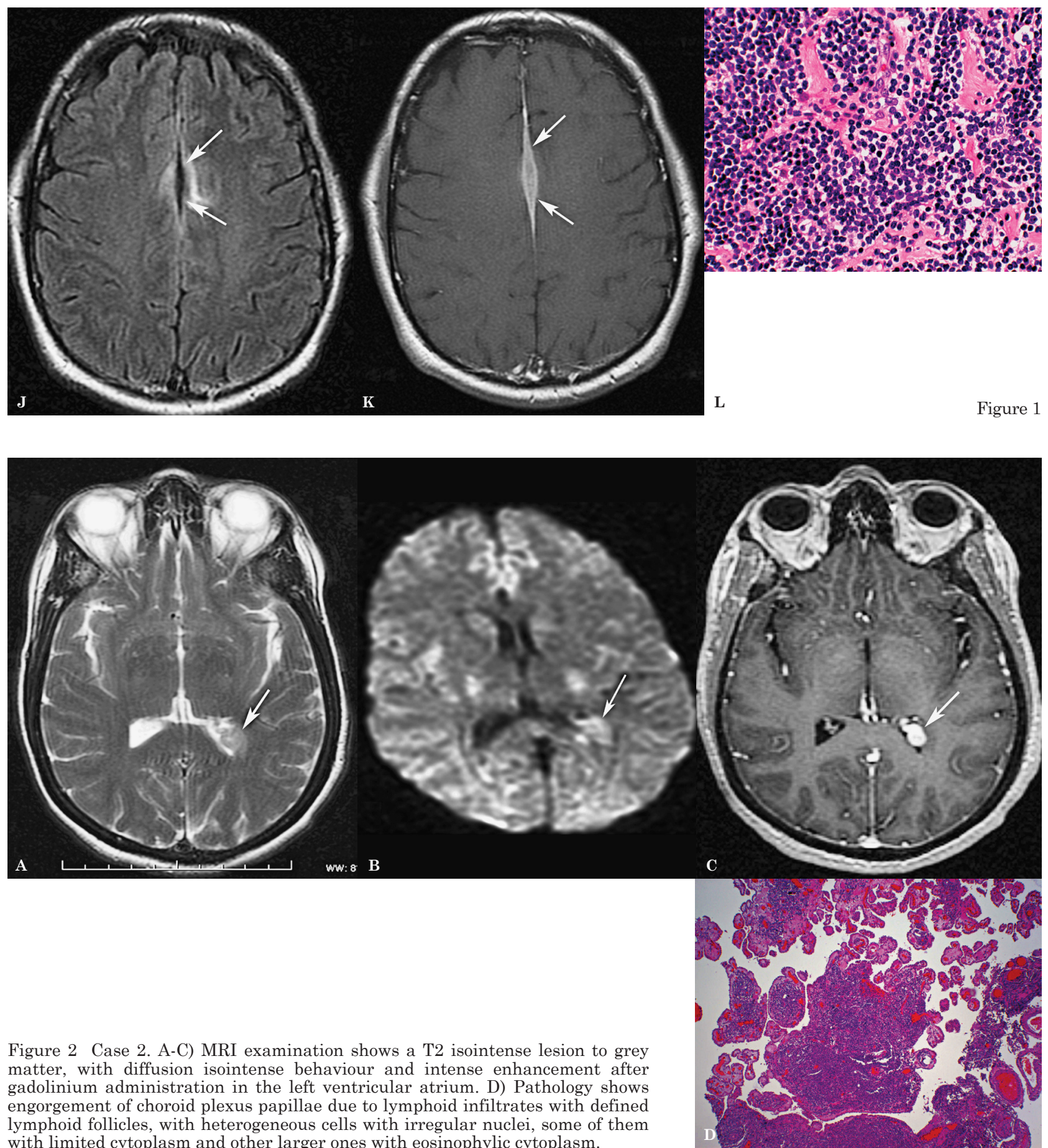

Figure 2 Case 2. A-C) MRI examination shows a T2 isointense lesion to grey matter, with diffusion isointense behaviour and intense enhancement after gadolinium administration in the left ventricular atrium. D) Pathology shows engorgement of choroid plexus papillae due to lymphoid infiltrates with defined lymphoid follicles, with heterogeneous cells with irregular nuclei, some of them with limited cytoplasm and other larger ones with eosinophylic cytoplasm.

Figure 1

lower extremity clonic movements progressing to right upper extremity involvement, with episodes lasting for about two minutes, responding to anti-seizure medication. Neurologic examination showed no focal deficits or asymmetry. Head CT scan without and with contrast (Figure 1A,B) showed an extra-axial lesion hy- perdense to brain along the interhemispheric fissure with mild mass effect and associated bilateral frontal subcortical vasogenic oedema. This lesion enhanced intensely with intravenous contrast on $\mathrm{CT}$.

Magnetic resonance imaging (MRI) evaluation showed a T1 and FLAIR isointense lesion 
to grey matter (Figure 1C,D) with T2 hypointense behaviour (Figure 1E) with perilesional oedema. The gadolinium-enhanced axial T1 image (Figure 1F) showed homogeneously intense enhancement. Proton MR Spectroscopy (Figure 1I) showed a significant elevation of the choline peak with a choline/creatine ratio larger than 1 , a mild decrease in N-acetyl-aspartate (NAA), and no lipid or lactate abnormalities. The diffusion-weighted image showed lesion isointensity (Figure 1G), while the apparent diffusion coefficient map showed moderate diffusion restriction compared to normal grey matter (Figure $1 \mathrm{H})$. The imaging findings initially favoured a lenticular falx meningioma, but the perilesional oedema, diffusion signal abnormality, diffusion restriction and MR spectroscopy suggested a more aggressive lesion, favouring a Grade II atypical meningioma. After initial treatment with antiepileptic drugs and corticosteroids, the follow-up MRI showed a significant reduction in perilesional oedema, with the lesion borders also reduced in size, becoming less defined (Figure $1 \mathrm{~J}, \mathrm{~K})$. In view of this response, granulomatous lesions and lymphoma were also added to the differential diagnosis.

The patient returned nine months later with a new episode of seizures, leading to uncomplicated surgical resection. Immuno-histochemistry demonstrated the presence of CD20 +, CD79a +, Bcl-2 +, IgD, CD 3, CD43, K y $\lambda$, Ki $67: 15 \%$. Molecular analysis was positive for monoclonality. The final result (Figure 1L) was type B cell MALT lymphoma with falx and adjacent brain infiltration.

\section{Case 2}

A 65-year-old woman was referred from another medical facility with a head CT incidental finding of a left ventricular atrium lesion, which enhanced with iodinated contrast.

MRI examination at our institution (Figure 2 A-C) showed a T2 isointense lesion to grey matter, with diffusion isointense behaviour and intense enhancement after gadolinium administration. MR perfusion and MR spectroscopy were non-contributory. No interval growth was perceived on follow-up examinations up to one year later, favouring an asymmetric but otherwise normal choroid glomus. The patient was afraid that she had a malignant tumour and insisted on tumour removal. Immuno-histochemistry demonstrated CD20 +, CD79a +, Bcl-2 +, CD43 +, CD3 y CD5, CD21,
CD23, Bcl-6 and CD10, IgD and Ki 67 15\%. Final pathology (Figure 2D) was a MALT lymphoma in the choroid plexus.

\section{Discussion}

Primary central nervous system (CNS) lymphomas represent $3-4 \%$ of primary brain tumours ${ }^{5}$. Large B-cell diffuse lymphomas are the most frequent subgroup, comprising $90 \%$ of all primary CNS lymphomas, most frequently seen in immune compromised patients as aggressive intraparenchymal tumours. These lesions very rarely involve the dura mater exclusively ${ }^{5}$. The marginal zone B-cell lymphomas, either primarily or secondarily involving the CNS, are very rare ${ }^{5-7}$.

Whenever MALT lymphomas involve the CNS, they most commonly involve the dura mater in comparison to other CNS compartments ${ }^{8}$. The anatomic location of dura mater involvement is variable, more frequent in hemispheric convexities, temporal-parietal and parietal-occipital dural surfaces, interhemispheric falx, tentorium, cavernous sinus and the cerebellopontine angle region ${ }^{9}$.

Dural involvement by lymphoma may present as a slow-growing dural mass with no apparent infiltration of adjacent brain parenchyma ${ }_{9,10}$ and with no systemic involvement. Most patients' clinical signs and symptoms are subtle and insidious, requiring long evaluations and follow-up before the diagnosis becomes clear 9. Most frequent symptoms include dizziness, fainting spells, nausea, vomiting, memory impairment and blurred vision ${ }^{9,10}$. They may in a few instances show a more rapid progression in a few weeks of evolution. MALT lymphomas are more frequent between 40 and 50 years of age, with a 5:1 female:male dominance ${ }^{10}$.

Dural-based MALT lymphomas are very similar to meningiomas on imaging ${ }^{10-12}$. They appear as a localized solid extra-axial lesion, either dural-based or leptomeningeal, frequently showing a "dural tail" sign on gadolinium-enhanced images, suggestive but not specific for meningioma. Although this sign is seen in approximately $60 \%$ of meningiomas, it can also be seen in other conditions affecting the dura, to include from post radiation secondary effects to post surgical dural reaction, neurosarcoidosis, aspergillosis, dural metastasis, chloromas or schwannomas. Lymphomas can also induce vasogenic oedema, mass effect and ventricular compression. 
The differential diagnosis of dural-based lesions is complex, requiring not just the imaging studies but clinical information regarding the patient's prior diseases ${ }^{13,14}$ to exclude metastases, secondary lymphoproliferative lesions, infection or inflammatory or granulomatous disease ${ }^{15-18}$ such as tuberculosis, syphilis, Wegener's disease or Rosai-Dorfman disease ${ }^{19,20}$.

Imaging findings suggesting aggressive biological behaviour are determinant in the differential considerations, such as strong and irregular enhancement or interval growth from prior studies. Atypical meningiomas (grade II) or malignant meningiomas (grade III) may also show aggressive imaging behaviour, becoming more invasive with higher grades ${ }^{21}$. The presence of lesion calcifications, adjacent skull hyperostosis and homogeneous enhancement favours benign meningioma (grade I) ${ }^{21,22}$.

The hyperintense diffusion signal abnormality with diffusion restriction associated with lesion high cellular packing is very relevant to diagnose atypical lesions ${ }^{23}$ since the most frequent benign meningiomas will not restrict diffusion, while lymphomas and other aggressive dural-based lesions will frequently do so. The transient "response" to corticosteroids in duralbased lesions may also favour the diagnosis of lymphoma over benign meningiomas ${ }^{24}$.

The second most frequent site of MALT lymphoma involvement intracranially is the glomus of the choroid plexus in the lateral ventricles, an ectodermal origin structure devoid of a blood brain barrier. Involvement by CT usually shows hyperdense asymmetrically bulky choroid plexus.

MRI behaviour is variable, T1 iso to hyperintense and T2 iso to hypointense, while gadolinium enhancement is invariably intense and homogeneous ${ }^{25,26}$. Larger lesions may trigger peritrigonal oedema and subependymal venous engorgement ${ }^{26}$.

Age of the patient at time of diagnosis impacts differential diagnosis. Children and young adults will favour ependymoma, central neurocytoma, subependymal giant cell astrocytoma and choroid plexus papilloma and carcinoma ${ }^{27}$. Middle-aged patients favour meningioma, lymphoma, choroid plexus papilloma, subependymoma and metastases ${ }^{27}$. Renal and pulmonary malignancies are the most frequent source of the rare choroid metastases. Meningiomas and choroid papillomas calcify most frequently, while hydrocephalus is a more frequent presenting feature in choroid plexus papilloma (overproduction of cerebrospinal fluid) and subependymoma (ventricular obstruction). Papillomas are more frequent in the atrium and may extend extraventricularly along the choroidal fissure while subependymoma rarely will behave extraventricularly, being most frequently a fourth ventricle wall lesion ${ }^{27}$.

The most frequent lesions localizing to the lateral ventricle are the meningiomas and the lymphomas ${ }^{27}$, with their differentiation by imaging still remaining very difficult. Diffusion sequences are more difficult to evaluate at this location, but when positive are very helpful. 


\section{References}

1 Eom KS, Park MR, Choi KH, et al. Gastric mucosaassociated lymphoid tissue lymphoma followed by primary central nervous system lymphoma. J Korean Neurosurg Soc. 2012; 51 (6): 377-379. doi: 10.3340/ jkns.2012.51.6.377.

2 Shaia J, Kerr PB, Saini A, et al. Mucosa-associated lymphoma tissue of the dura presenting as meningioma. South Med J. 2010; 103 (9): 950-952. doi: 10.1097/ SMJ.0b013e3181eb3477.

3 Almousa R, Nga ME, Sundar G. Marginal zone B-cell orbital lymphoma with intracranial involvement. Ophthal Plast Reconstr Surg. 2010; 26 (3): 205-206. doi: 10.1097/IOP.0b013e3181b8e0bf.

4 Eckardt AM, Lemound J, Rana M, et al. Orbital lymphoma: diagnostic approach and treatment outcome. World J Surg Oncol. 2013; 11: 73. doi: 10.1186/14777819-11-73.

5 Ueba T, Okawa M, Abe H, et al. Central nervous system marginal zone B-cell lymphoma of mucosa-associated lymphoid tissue type involving the brain and spinal cord parenchyma. Neuropathology. 2013; 33 (3): 306-311. doi: 10.1111/j.1440-1789.2012.01350.x.

6 Fadugba O, Nguyen TT, McGill J, et al. De novo large B-cell lymphoma in a parenchymal brain lesion: evidence of clonal evolution from splenic marginal zone lymphoma. J Clin Oncol. 2011; 29 (9): e234-236. doi: 10.1200/JCO.2010.32.5811.

7 Ponzoni M, Bonetti F, Poliani LP, et al. Central nervous system marginal zone B-cell lymphoma associated with Chlamydophila psittaci infection. Hum Pathol. 2011; 42 (5): 738-742. doi: 10.1016/j.humpath.2010.08.014.

8 Papanicolau-Sengos A, Wang-Rodriguez J, Wang HY, et al. Rare case of a primary non-dural central nervous system low grade B-cell lymphoma and literature review. Int J Clin Exp Pathol. 2012; 5 (1): 89-95.

9 Razag, W, Goel A, Amin A, et al. Primary central nervous system mucosa-associated lymphoid tissue lymphoma: case report and literature review. Clin Lymphoma Myeloma. 2009; 9 (3): E5-9. doi: 10.3816/ CLM.2009.n.052.

10 Bayraktar S, Stefanovic A, Montangue N, et al. Central nervous system manifestations of marginal zone B-cell lymphoma. Ann Hematol. 2010; 89 (10): 10031009. doi: 10.1007/s00277-010-0976-3.

11 Park I, Hugh J, Kim HJ, et al. Primary central nervous system marginal zone B-cell lymphoma of the basal ganglia mimicking low-grade glioma: a case report and review of the literature. Clin Lymphoma Myeloma. 2008; 8 (5): 305-308. doi: 10.3816/CLM.2008.n.043.

12 Kulkarni KM, Sternau L, Dubovy SR, et al. Primary dural lymphoma masquerading as a meningioma. J Neuroophtalmol. 2012; 32 (3): 240-242. doi: 10.1097/ WNO.0b013e31825103a5.

13 Sahin F, Saydam G, Ertan Y, et al. Dural plasmacytoma mimicking meningioma in a patient with multiple myeloma. J Clin Neurosci. 2006; 13 (2): 259-261. doi: 10.1016/j.jocn.2005.03.033.

14 Richiello A, Sparano L, Del Basso DE, et al. Dural metastasis mimicking falx meningioma. Case report. J Neurosurg Sci. 2003; 47 (3): 167-171.

15 Loy ST, Tan CWT. Granulomatosis meningitis. Singapore Med J. 2009; 50 (11): e371-373.

16 Schmidt S, Eich G, Geoffray A, et al. Extraosseous langerhans cell histiocitosis in children. Radiographics. 2008; 28 (3): 707-726. doi: 10.1148/rg.283075108.

17 Segal BM. Neurosarcoidosis: diagnostic approaches and therapeutic strategies. Curr Opin Neurol. 2013; 26 (3): 307-313. doi: 10.1097/WCO.0b013e3283608459.

18 Gupta RK, Kumar S. Central Nervous System tuberculosis. Neuroimaging Clin N Am. 2011; 21 (4): 795-814. doi: 10.1016/j.nic.2011.07.004.

19 Wahba M. Case report of intracranial rosai-dorfman disease. Hosp Pract (1995). 2013; 41 (4): 83-86. doi: 10.3810/hp.2013.10.1085.

20 Udono H, Fukuyama K, Okamoto H, et al. Rosai-Dorfman disease presenting multiple intracranial lesions with unique findings on magnetic resonance imaging. Case report. J Neurosurg. 1999; 91 (2): 335-339. doi: 10.3171/jns.1999.91.2.0335.

21 Buetow MP, Buetow PC, Smirniotopoulos JG. From the archives of the AFIP. Typical, atypical, and misleading features in meningioma. Radiographics. 1991; 11: 1087-1106. doi: 10.1148/radiographics.11.6.1749851.

22 Sacho RH, Kogels M, Plessis DD, et al. Primary diffuse large B-cell cetral nervous system lymphoma presenting as an acute space-occupying subdural mass. J Neurosurg. 2010; 113 (2): 384-387. doi: 10.3171/2010.2.JNS091554.

23 Fatima Z, Motosugi U, Wagar AB, et al. Associations among q-space MRI, diffusion-weighted MRI and histopathological parameters in meningiomas. Eur Radiol. 2013; 23 (8): 2258-2263. doi: 10.1007/s00330-013-28230 .

24 Matmati K, Matmati N, Hannum YA, et al. Dural MALT lymphoma with disseminated disease. Hematol Rep. 2010; 2 (1): e10.

25 Kelley TW, Prayson RA, Barnett GH, et al. Extranodal marginal zone B-cell lymphoma of mucosa-associated lymphoid tissue arising in the lateral ventricle. Leuk Lymphoma 2005; 46 (10): 1423-1427. doi: 10.1080/10428190500205895.

26 Jung TY, Jung S, Lee MC, et al. Extranodal marginal zone B-cell lymphoma mimicking meningioma in lateral ventricle: a case report and possible pathogenesis. J Neurooncol. 2006; 80 (1): 63-67. doi: 10.1007/s11060006-9153-x.

27 Koeller KK, Sandberg GD. From the archives of the AFIP. Cerebral intraventricular neoplasms: Radiologic-Pathologic correlation. Radiographics. 2002; 22 (6): 1473-1505. doi: 10.1148/rg.226025118.
Miss Cristina Sebastián Department of Radiology Universitary Hospital Miguel Server Isabel La católica, $\mathrm{n}^{\circ}$ 1-3 500009 Zaragoza, Spain E-mail: crissebseb@gmail.com 